\title{
Charakteristika eines finanz-dominierten Akkumulationsregimes in Europa
}

Engelbert Stockhammer

Die Beziehungen zwischen dem realen Sektor und dem Finanzsektor sind im Umbruch. Heftige Zyklen auf den Aktienmärkten und Wechselkurskrisen sind dafür die augenfälligsten Beispiele. Ökonomisch ebenso wichtig sind die zunehmende Verschuldung privater Haushalte und die Shareholder Value-Orientierung bei produzierenden Unternehmen. Welche Muster weist nun ein von der „Finanzialisierung" geprägtes Wirtschaftssystem auf? Und was bedeutet dies für Wachstum und wirtschaftliche Stabilität?

\section{Einleitung}

Der Begriff „Finanzialisierung“ umfasst eine Vielzahl heterogener Phänomene: die Deregulierung des Finanzsektors und die Entwicklung neuer Finanzinstrumente; die Liberalisierung der internationalen Kapitalströme und die zunehmende Instabilität der Wechselkurse; das Emporkommen institutioneller Investoren als neue, mächtige Akteure sowie heftige Zyklen auf den Aktienmärkten; „Shareholder Value“-Orientierung und Veränderungen in der Corporate Governance; verbesserter Zugang zu Krediten für soziale Gruppen, die einst als „underbanked“ bezeichnet worden sind; und das im Vergleich zur Nachkriegszeit hohe Niveau der Realzinsen. Der Begriff "Finanzialisierung" wurde auch verwendet, um Veränderungen in psychologischen und ideologischen Strukturen zu beschreiben. In diesem Beitrag wird der Versuch unternommen, mithilfe des regulationstheoretischen Begriffs „Akkumulationsregime" die makroökonomische Bedeutung dieser Veränderungen zu analysieren.

Die übliche keynesianische Nachfragefunktion wird diese Analyse strukturieren. Die aggregierte Nachfrage besteht aus dem privaten Konsum $(\mathrm{C})$, den Investitionen (I), den Nettoexporten (NX) und den Staatsausgaben $(\mathrm{G})$ des öffentlichen Sektors: $Y=C+I+N X+G .{ }^{1}$ Für jede dieser Komponenten soll untersucht werden, welche Veränderungen aufgetreten sind und wie weit sie mit Finanzialisierung in $\mathrm{Zu}-$ sammenhang gebracht werden können. Dies bedeutet auch, dass unterschiedliche Aspekte der Finanzialisierung bei der Analyse der verschiedenen makroökonomischen Aggregate relevant werden. Diese scheinbare Inkonsistenz ist jedoch nicht als
Schwäche dieses Artikels, sondern als Reflexion des derzeitigen Forschungsstands zur Finanzialisierung zu verstehen.

Neben der Frage, ob die makroökonomischen Verhaltensfunktionen durch Finanzialisierung verändert wurden, soll auch untersucht werden, ob diese Veränderungen zu einer kohärenten Struktur, eben einem Akkumulationsregime, geführt haben. Anders ausgedrückt wird der Frage nachgegangen, ob es sinnvoll ist, von einem finanz-dominierten Akkumulationsregime zu sprechen und, falls ja, was seine Charakteristika sind. Die Arbeit benutzt also den Rahmen der französischen Regulationstheorie, ${ }^{2}$ welche die makroökonomische Dynamik (eben das „Akkumulationsregime“) in ein bestimmtes institutionelles Gefüge (die „Regulationsweise") eingebettet sieht. Während es in der Regulationstheorie einen breiten Konsens darüber gibt, dass das fordistische Akkumulationsregime im Laufe der 1970er Jahre in die Krise gekommen ist, ist die Charakterisierung des post-fordistischen Regimes, ja selbst die Frage, ob ein solches bereits existiert, umstritten. Nachdem in der Analyse des post-fordistischen Regimes zunächst der Flexibilität (in Arbeitsverhältnissen und Produktion) und dann den Informations- und Kommunikationstechnologien eine prägende Rolle zugeschrieben wurde, haben die Veränderungen im Finanzsektor erst kürzlich mehr Beachtung gefunden. Der Begriff „finanz-dominiertes Akkumulationsregime" wird hier verwendet, um aufzuzeigen, dass finanzielle Entwicklungen die Struktur und Geschwindigkeit der Akkumulation prägen.

Der Begriff finanz-dominiert (financedominated) wird dabei in bewusster Abgrenzung zum Begriff finanz-getrieben (finance-led) genutzt, um deutlich zu machen, dass die Finanzialisierung die Muster der Akkumulation, d.h. die Zusammenset- zung der Nachfrage und ihre Volatilität, prägt. Boyer (2000) hatte in einem wichtigen Beitrag ein Akkumulationsregime als finanz-getrieben definiert, wenn ein Anstieg der Mindestrendite (der financial norm), die von den Finanzmärkten für reale Investitionsprojekte gesetzt wird, zu einem Anstieg des Wachstums führt. Diese Konzeption wird hier nicht verwendet. Der Begriff des finanz-dominierten Regimes ist vielmehr so definiert, dass Finanzialisierung das Wachstum positiv oder negativ beeinflussen kann. Es ist daher möglich, dass die makroökonomische Nachfrage nicht finanz-getrieben, aber dennoch strukturell vom Finanzsektor geprägt ist.

Der Übersichtlichkeit halber werden zunächst die Charakteristika des finanzdominierten Akkumulationsregimes zusammengefasst:

\footnotetext{
1 Statt als Nachfrage- oder Ausgabenfunktion kann die obige Gleichung auch als volkswirtschaftliche Sparrestriktion reformuliert werden. Das Sparen der Haushalte $\left(\mathrm{S}_{\mathrm{HH}}\right)$, der Unternehmen $\left(\mathrm{S}_{\mathrm{C}}\right)$, des Staates $\left(S_{G}\right)$ und des Außensektors $\left(S_{F}\right)$ müssen sich gegenseitig ausgleichen: $\mathrm{S}_{\mathrm{HH}}+\mathrm{S}_{\mathrm{C}}+\mathrm{S}_{\mathrm{G}}+\mathrm{S}_{\mathrm{F}}$ $=0$. Für jeden Schuldner muss es einen Gläubiger geben. Die Ersparnisse des Außensektors sind definitionsgemäß gleich den Nettokapitalzuflüssen. Die Kapitalflüsse werden in Abschnitt 4 diskutiert.

2 Die klassischen Arbeiten der (französischen) Regulationstheorie sind Aglietta (1979), Lipietz (1985) und Boyer (1990). Ähnlichkeiten zwischen der Regulationstheorie und dem (amerikanischen) Social-Structures-of-Accumulation-Ansatz (Gordon et al. 1982; Bowles et al. 1983) sind nun weithin anerkannt (z. B. McDonough/Nardone 2006).
}

Engelbert Stockhammer, Ass. Prof. Dr., Wirtschaftsuniversität Wien. Arbeitsschwerpunkte: Makroökonomie, Arbeitslosigkeit, Europäische Integration. e-mail: engelbert.stockhammer@wu-wien.ac.at 
- Da private Haushalte vermehrt Zugang $\mathrm{zu}$ Krediten bekommen und sich auch tatsächlich beträchtlich verschulden, werden die Konsumausgaben oft zum Konjunkturmotor. Da die angehäuften Schulden aber auch bedient werden müssen, stellt dies andererseits eine Quelle für potenzielle Instabilitäten und Krisen dar.

- Die Investitionsausgaben wachsen aufgrund der "Shareholder Value“-Orientierung und zunehmender Unsicherheit nur verhalten. Bemerkenswerterweise führen die hohen Profite nicht zu einem entsprechenden Anstieg der Investitionen.

- Die Deregulierung der Finanzmärkte führt zu einem Anstieg der Kapitalströme und, in Konsequenz, zu einer zunehmenden Volatilität der Wechselkurse, was sich in einer erhöhten Unsicherheit und einer Häufung schwerer Wechselkurskrisen niederschlägt.

- Trotz der neoliberalen Attacke sind die Staatsquoten hoch geblieben und tragen so zur Stabilisierung der Wirtschaft bei.

- Insgesamt ist das finanz-dominierte Akkumulationsregime daher durch niedrige längerfristige Wachstumsraten und häufige Krisen, die oft in Wechselkurskrisen ihren Ausgangspunkt haben, gekennzeichnet.

Dieser Beitrag unterscheidet sich in zweierlei Hinsicht von anderen Diskussionen der Finanzialisierung. Erstens wird ein weiter Begriff der Finanzialisierung verwendet. Makroökonomische Ansätze (Boyer 2000; Stockhammer 2004a, 2005/06; Hein/ van Treeck 2007; Skott/Ryoo 2007) gebrauchen zumeist einen engeren Begriff, der analytisch besser handhabbar ist, jedoch der Vielfalt der Veränderungen nicht gerecht wird. Zweitens richtet dieser Artikel seinen Fokus auf westeuropäische Länder. Ein großer Teil der empirischen Literatur behandelt, nicht zuletzt aus Gründen der Datenverfügbarkeit, die USA (Duménil/ Lévy 2001; Crotty 2003; Krippner 2005). Da Finanzialisierung unterschiedliche Auswirkungen auf verschiedene Länder bzw. Ländergruppen haben kann, ist eine Diskussion europäischer Entwicklungen von besonderem Interesse. Die europäische Erfahrung ist dabei geprägt durch eine bestimmte, neoliberale Form der wirtschaftspolitischen Integration (Bieler 2003).

\section{Der Hintergrund: Eine neoliberale Regulationsweise}

Finanzialisierung wurde ermöglicht durch die Deregulierung von nationalen und internationalen Finanzmärkten, die das Ergebnis von politischen Entscheidungen ist. Vor einer Diskussion des Akkumulationsregimes ist daher kurz auf die (wirtschaftspolitische) Regulationsweise einzugehen. Aus Platzgründen können aber nur einige Entwicklungen in der neoliberalen Regulationsweise in Europa knapp thematisiert werden:

- Machtverschiebung von Arbeit zu Kapital. Diese findet in fallenden Lohnquoten ihren Ausdruck. Die Ursachen sind vielfältig. Zu nennen wären der sinkende Organisationsgrad der Gewerkschaften, die demoralisierende Wirkung der Arbeitslosigkeit und das höhere Drohpotenzial des Kapitals durch die Möglichkeit von Produktionsverlagerungen.

- Redefinition der Rolle des Staates. Dies beinhaltet Privatisierungen und Deregulierungen. Deregulierungen auf den Finanzmärkten sind dabei nur ein, hier aber offensichtlich zentraler Bereich. Auf die Größe des Staatssektors wird später noch eingegangen.

- Änderung der Geldpolitik. Von der akkomodierenden Politik der Nachkriegszeit, die (im fordistisch-keynesianischen Regime) zur Sicherung der Vollbeschäftigung beitrug, fand eine Verschiebung der Prioritäten hin zu Preisstabilität auch auf Kosten von Massenarbeitslosigkeit statt.

Der Neoliberalismus manifestierte sich in politischen Entscheidungen, die in Kontinentaleuropa oft auf der europäischen statt der nationalstaatlichen Ebene gefällt wurden. ${ }^{3}$ Der Maastricht Vertrag, der Stabilitäts- und Wachstumspakt und die Dienstleistungsrichtlinie sind hier nur die prominentesten Beispiele. Die EU ist dabei aber kein homogener Akteur. Die Entscheidungsgremien sind Schauplatz für Richtungskämpfe, wobei nunmehr eine stark neoliberale Orientierung dominiert. Van Apeldoorn (1999) verwendet den Begriff des eingebetteten Neoliberalismus (embedded neoliberalism), um die hegemoniale Struktur zu beschreiben. Neoliberalis- mus bedeutet aber nicht eine generelle Flexibilisierung, sondern eine selektive. So wurden die Regeln für Fiskalpolitik stringenter, und die Wechselkurse wurden im Rahmen des Euro-Systems eingefroren.

\section{Veränderungen im Konsumverhalten?}

Veränderungen im Konsumverhalten sind von besonderem Interesse, da die Konsumausgaben die größte Komponente der aggregierten Nachfrage darstellen. Hier stehen zwei miteinander verbundene Fragen im Vordergrund. Erstens: In welchem Ausmaß reagieren private Konsumausgaben auf eine Veränderung der Vermögenspositionen der Haushalte? Und zweitens: In welchem Ausmaß haben Haushalte neue Möglichkeiten der Verschuldung genutzt, um damit Konsumausgaben zu finanzieren?

Bekanntermaßen wurde die Konjunkturentwicklung in den USA in Phasen der 1990er Jahre und in den frühen 2000er Jahren stark von der Dynamik der Konsumausgaben angetrieben. Um diese Entwicklung zu erklären, griffen die Makroökonomen auf die Theorie zurück, dass nicht das laufende Einkommen, sondern die Vermögenspositionen die Konsumausgaben bestimmen. Zunächst wurde der Boom auf den Aktienmärkten als Erklärung herangezogen (Boone et al. 1998). Als es mit dem Platzen der Aktienblase jedoch zu keinem Einbruch der Konsumausgaben kam, rückten die Immobilienpreise in den Mittelpunkt des Interesses. Etliche Studien fanden deutlich höhere Konsumneigungen aus Immobilienvermögen als aus Finanzvermögen (Catte et al. 2004; Girouard et al. 2006; Case et al. 2001). Für europäische Länder sind die geschätzten Effekte jedoch zumeist wesentlich geringer als für die USA. Darüber hinaus ist der Hausbesitz hier oft nicht so verbreitet und die Datenlage dünn.

Ein zweiter Aspekt der Finanzialisierung ist, dass Haushalte nunmehr besseren

\footnotetext{
Während die Regulationstheorie in der Analyse des Fordismus einen Fokus auf nationale Regulationsweisen richtete, wird in der Diskussion um den Post-Fordismus oft die zentrale Rolle von supra-nationalen Akteuren und Strukturen hervorgehoben (Dannreuther/Petit 2006).
} 


\begin{tabular}{|c|c|c|c|c|c|c|c|}
\hline & \multicolumn{4}{|c|}{$\begin{array}{l}\text { 1.1 Private Konsumausgaben } \\
\text { (Durchschnitt) }\end{array}$} & \multicolumn{3}{|c|}{$\begin{array}{l}1.2 \text { Schulden der privaten } \\
\text { Haushalte }\end{array}$} \\
\hline & $1970 \mathrm{er}$ & $1980 \mathrm{er}$ & $1990 \mathrm{er}$ & $2000 \mathrm{er}$ & 1995 & 2000 & 2005 \\
\hline Belgien & 63 & 67 & 63 & 63 & - & - & - \\
\hline Dänemark & 63 & 65 & 62 & 59 & 188 & 236 & 2601) \\
\hline Deutschland & 64 & 66 & 69 & 70 & 97 & 111 & 107 \\
\hline Griechenland & 63 & 70 & 75 & 74 & - & - & - \\
\hline Spanien & 72 & 73 & 70 & 70 & 59 & 83 & 1071) \\
\hline Frankreich & 63 & 66 & 65 & 66 & 66 & 78 & 89 \\
\hline Irland & 73 & 75 & 68 & 62 & & 81 & 141 \\
\hline Italien & 67 & 68 & 69 & 70 & 32 & 46 & 59 \\
\hline Luxemburg & 64 & 59 & 55 & 53 & - & - & - \\
\hline Niederlande & 59 & 60 & 59 & 58 & 113 & 175 & 246 \\
\hline Österreich & 66 & 68 & 68 & 67 & - & - & - \\
\hline Portugal & 79 & 75 & 74 & 78 & - & - & - \\
\hline Finnland & 64 & 65 & 66 & 61 & 64 & 66 & 89 \\
\hline Schweden & 56 & 57 & 59 & 56 & 90 & 107 & 134 \\
\hline Vereinigtes Königreich & 69 & 71 & 73 & 72 & 106 & 118 & 159 \\
\hline$E U-12^{2)}$ & 66 & 67 & 66 & 65 & 91 & 110 & 139 \\
\hline USA & 70 & 73 & 76 & 80 & 93 & 107 & 135 \\
\hline Japan & 61 & 64 & 67 & 71 & 113 & 136 & 1321) \\
\hline
\end{tabular}

Zugang zu Krediten haben als zuvor. Diese können in Form von Hypothekarkrediten, Konsumentenkrediten oder Kreditkartenschulden wahrgenommen werden und gehen teils auf das aggressive Marketing der Banken zurück. Als Folge dieser Entwicklung ist die Verschuldung der Haushalte angestiegen. ${ }^{4}$ Daten dazu sind jedoch nicht standardisiert verfügbar und daher international schwer vergleichbar. Tabelle 1 beruht auf OECD-Schätzungen (Girouard et al. 2006). Die europäischen Länder weisen unterschiedliche Werte auf. Jedoch sind die Verschuldungsquoten in allen Ländern angestiegen und die Durchschnittswerte sind mit jenen der USA vergleichbar.

Anders als die USA (und Japan) weisen die europäischen Länder keinen Anstieg der Konsumquote auf. Während die Konsumquote sich in den USA (und Japan) seit den 1970er Jahren erhöht, stagniert sie in Europa. ${ }^{5}$ Nur Griechenland weist einen starken Anstieg auf. Es mag also zunächst so aussehen, als habe die zunehmende Verschuldung keinen Effekt auf die Konsumausgaben. Dies wäre jedoch ein voreiliger Schluss. Erstens ist in Europa die Lohnquote seit 1980 drastisch gefallen. Da Lohneinkommen typischerweise höhere Konsumneigungen aufweisen als Profiteinkommen, hätte dies zu einem Fall der Konsumquoten führen müssen. Stockhammer et al. (2007) finden für den Euro-Raum ein
Konsumdifferential zwischen dem Konsum Lohneinkommen und dem aus Profiteinkommen von ca. $0,4 .{ }^{6}$ Bei einem Fall der Lohnquote um 10 Prozentpunkte würde dies einem Rückgang der Konsumquote um ca. 4 Prozentpunkte entsprechen. Die steigenden Schulden der privaten Haushalte könnten also zur Kompensierung der Verlangsamung des Lohnwachstums verwendet worden sein. Zweitens könnten die steigenden Haushaltsschulden natürlich auch in den Hausbau statt in den Konsum geflossen sein. Dies wird im nächsten $\mathrm{Ab}$ -

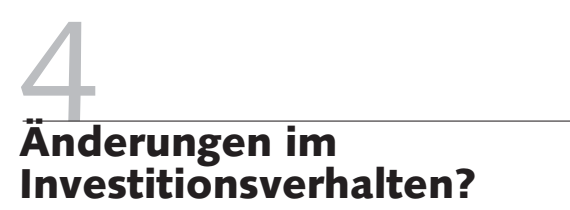

Die Finanzialisierung ging einher mit vielen Veränderungen, die für das Investitionsverhalten relevant sein können. So hat die "Shareholder Value“-Orientierung die Managementziele verändert und die Instabilität der Finanzmärkte hat dazu geführt, dass die Firmen nun einem höheren Grat an Unsicherheit ausgesetzt sind. Gleichzeitig bieten neue Finanzinstrumente Versicherungsmöglichkeiten gegen Wechselkursschwankungen, und das Wachstum der Finanzmärkte könnte die Investitionsfinanzierung vereinfachen. Es ist jedoch schnitt untersucht. schwierig, die Bedeutung dieser Entwicklung für die Investitionen empirisch zu belegen, was auch daran liegt, dass die Investitionen selbst eine ohnehin schwer erklärbare Variable sind.

Tabelle 2 fasst die Entwicklung der Investitionstätigkeit relativ zu den Profiten zusammen. Im EU-Durchschnitt ist der Anteil der Investitionen an den Profiten von $47 \%$ (in den 1970er Jahren) auf $44 \%$ (in den 1990er Jahren und danach) gefallen. Besonders ausgeprägt war dieser Rückgang in Deutschland, aber auch Frankreich, das Vereinigte Königreich (VK) und die USA weisen einen fallenden Verlauf auf. Nur in wenigen Ländern, wie Griechenland und Spanien, wird im Verhältnis zu den Profiten mehr investiert.

Für diese Entwicklung gibt es mehrere Erklärungen, die direkt mit der Finanzialisierung zu tun haben. Erstens beinhaltet das in der Volkswirtschaftlichen Gesamtrechnung (und hier) verwendete Maß für Profite die Zinszahlungen. Bei steigenden Zinsen können den Unternehmen trotz hoher Gewinne geringe Mittel zum investieren bleiben (Duménil/Lévy 2001; Crotty 2003). Eine Bereinigung um die Zinszahlungen ist aus Datengründen schwierig.

Zweitens gab es eine Änderung in der Ausrichtung des Managements. Nicht mehr die Arbeitsbeziehungen (wie zur Zeit des Fordismus) stehen heute im Zentrum,

\footnotetext{
4 In der Mainstreamliteratur wird angenommen, dass die steigende Verschuldung der Haushalte rational ist. Die Verschuldung steigt, weil das Vermögen wächst. Es ist aber auch möglich, dass ein wesentlicher Teil der Schulden Ergebnis eines irrationalen Verhaltens ist, da viele Konsumenten noch nicht gelernt haben, mit den neuen Möglichkeiten, sich zu verschulden, umzugehen. Aus der experimentellen Psychologie ist bekannt, dass die Zahlungsmethode die Kaufbereitschaft beeinflusst: Typischerweise sitzt das Geld lockerer, wenn Kreditkarten verwendet werden

5 In Tabelle 1 und den folgenden Tabellen werden die Daten in 10-Jahresmittelwerten angegeben. Die 1970er Jahre können als die fordistische Phase betrachtet werden, auch wenn sich das Ende des Fordismus nicht exakt terminieren lässt. Danach beginnt die Phase des Neoliberalismus. In Europa waren die 1980er Jahre durch das europäische Währungssystem mit der DM als Leitwährung und die 1990er Jahre durch den Binnenmarkt und den Stabilitäts- und Wachstumspakt gekennzeichnet. Die Jahre nach 2000 stellen einen kürzeren Zeitraum dar und sollten daher mit Vorsicht interpretiert werden.

6 Dieser Wert liegt in einer ähnlichen Größenordnung wie der für andere Länder (Naastepad/Storm 2006/07; Hein/Vogel 2007).
} 
sondern die Shareholder. Mehrere Studien zu den Effekten der "Shareholder Value“Orienterung haben gezeigt, dass diese zu Beschäftigungsabbau und Aktienrückkäufen, aber nicht zu mehr Investitionen führt (Lazonick/O'Sullivan 2000; Stockhammer 2004a). Stockhammer (2004a) findet in Schätzungen heraus, dass die Finanzialisierung, welche anhand der Finanzinvestitionen der nicht-finanziellen Unternehmungen (bzw. der Einkommen daraus) gemessen wird, einen wesentlichen Teil des Rückgangs im Wachstum des Kapitalstocks erklärt, wenn auch mit großen Unterschieden zwischen einzelnen Ländern.

Drittens führte die Deregulierung der Finanzmärkte zu einer Erhöhung der Unsicherheit, was Investitionsausgaben, die oft kaum reversibel sind, weniger attraktiv macht. Speziell die Volatilität der Wechselkurse scheint einen negativen Einfluss auf die Investitionstätigkeit auszuüben (Carruth et al. 2000). Da Unsicherheit definitionsgemäß schwer messbar ist, ist auch hier eine exakte Bestimmung der Größe des Effekts sehr schwierig.

Insgesamt scheinen die Unternehmen die neuen Finanzinstrumente nicht zur Investitionsfinanzierung zu nutzen. Speziell für die angelsächsischen Länder ist belegt, dass Aktienrückkäufe beträchtliche Mittel gebunden haben (Schaberg 1999). Auch scheinen die Unternehmen wenig Fremdkapital aufzunehmen und sich eher in Netto-Gläubiger zu verwandeln, was einer Umkehr ihrer traditionellen Rolle im volkswirtschaftlichen Kreislauf gleichkäme. $^{7}$

Abschließend ist noch kurz auf die Rolle der Wohnungsbauten innerhalb der gesamtwirtschaftlichen Investitionen einzugehen. Wie bereits diskutiert, hat die Verschuldung der privaten Haushalte zugenommen. Es wäre möglich, dass damit Bauinvestitionen finanziert wurden. Wie Tabelle 2, Spalte 2 zeigt, ist aber der Anteil des Wohnungsbaus an den Gesamtinvestitionen im Durchschnitt der EU (und auch in den USA) gesunken. Im ungewichteten Durchschnitt der EU-Staaten sinken die Bauinvestitionen von $86 \%$ (in den 1970ern) auf $50 \%$ (in den 1990er Jahren) der Gesamtinvestitionen. Bloß in Irland ist ein kräftiger Anstieg feststellbar.

Insgesamt finden wir also keine Evidenz dafür, dass die Finanzialisierung die Investitionen in Europa belebt hätte. Vermutlich haben höhere Zinsen, die Orientierung am "Shareholder Value“ und die

Tabelle 2: Investitionen - in \%-

\begin{tabular}{|c|c|c|c|c|c|c|c|c|}
\hline & \multicolumn{4}{|c|}{$\begin{array}{l}2.1 \text { Investitionen in \% des } \\
\text { Betriebsüberschusses (Durchschnitt) }\end{array}$} & \multicolumn{4}{|c|}{$\begin{array}{l}\text { 2.2 Wohnungsbauinvestionen in \% } \\
\text { der Investitionen (Durchschnitt) }\end{array}$} \\
\hline & $1970 \mathrm{er}$ & $1980 \mathrm{er}$ & 1990er & $2000 \mathrm{er}$ & $1970 \mathrm{er}$ & $1980 \mathrm{er}$ & $1990 \mathrm{er}$ & $2000 \mathrm{er}$ \\
\hline Österreich & 59 & 50 & 47 & 44 & 59 & 52 & 44 & 29 \\
\hline Belgien & & & & & 65 & 38 & 38 & 35 \\
\hline Dänemark & 46 & 47 & 46 & 49 & 171 & 61 & 33 & 35 \\
\hline Finnland & 57 & 57 & 41 & 36 & 67 & 55 & 48 & 44 \\
\hline Frankreich & 46 & 46 & 42 & 43 & 78 & 63 & 47 & 43 \\
\hline Deutschland & 52 & 48 & 42 & 35 & - & - & 60 & 50 \\
\hline Griechenland & 24 & 24 & 26 & 36 & 105 & 86 & 52 & 29 \\
\hline Irland & 50 & 44 & 30 & 28 & 94 & 92 & 94 & 111 \\
\hline Italien & 41 & 36 & 31 & 33 & - & - & - & - \\
\hline Luxemburg & 39 & 48 & 51 & 50 & - & - & - & - \\
\hline Niederlande & 48 & 39 & 38 & 38 & 76 & 63 & 54 & 52 \\
\hline Portugal & 37 & 35 & 31 & 34 & - & - & - & - \\
\hline Spanien & 47 & 40 & 44 & 47 & 67 & 55 & 43 & 40 \\
\hline Schweden & 59 & 52 & 46 & 51 & 79 & 60 & 32 & 17 \\
\hline Vereinigtes Königreich & 55 & 48 & 44 & 42 & 86 & 82 & 60 & 47 \\
\hline$E U-12^{1)}$ & 47 & 44 & 40 & 40 & 86 & 64 & 50 & 44 \\
\hline USA & 46 & 44 & 39 & 39 & 81 & 55 & 47 & 44 \\
\hline Japan & 58 & 59 & 61 & 56 & 60 & 41 & 33 & 26 \\
\hline
\end{tabular}

Zunahme der Unsicherheit kräftig zum Rückgang der Investitionen in Relation zu den Profiten beigetragen. Auch der Aktienboom hatte kaum positive Auswirkungen auf die Investitionen. Üblicherweise finden ökonometrische Studien, dass Aktienkurse (selbst wenn sie steigen) kaum Auswirkungen auf die Investitionstätigkeit haben (Chirinko 1993; Ford/Poret 1991).

\section{Außenhandel und Kapitalflüsse}

In der aggregierten Nachfragefunktion ist der Außenbeitrag über die Nettoexporte repräsentiert, definiert als Exporte minus Importe. Die Nettoexporte müssen (abgesehen von den Veränderungen der Reserven der Zentralbank) den Kapitalexporten entsprechen. Es ist dieser zweite Aspekt, die Kapitalbilanz, der im Zusammenhang mit Finanzialisierung von Interesse ist, da die Kapitalflüsse durch die Deregulierung der Finanzmärkte stark zugenommen haben.

Die schwersten Wirtschaftskrisen gingen in den letzten beiden Jahrzehnten von Devisenmärkten aus: Mexiko 1994, Türkei 1994 und 2001, Korea und Malaysia im Laufe der Südost-Asien Krise 1997/98 und Argentinien 2001 sind die prominentesten Beispiele für heftige Wirtschaftskrisen, ${ }^{8}$ die durch abrupte Änderungen der Wechsel- kurse ausgelöst wurden, welche auf kurzfristige internationale Kapitalbewegungen zurückzuführen sind. In Europa erlebte vor allem Schweden nach den Turbulenzen im Europäischen Währungssystem (EWS) 1992/93 eine langwierige Krise.

Der Grund, warum die Wechselkurskrisen so starke Auswirkungen haben, ist, dass in liberalisierten Finanzmärkten Zinsarbitragegeschäfte (heute oft carry trade genannt) sehr attraktiv sind. Sind die Zinsen (bei gegebenen Wechselkursen) in Europa niedriger als z. B. in der Türkei, so ist es verlockend, Schulden in Euro aufzunehmen und damit türkische Staatspapiere zu kaufen. Soll- und Haben-Positionen bestehen dann in unterschiedlichen Währungen und eine Änderung des Wechselkurses führt dann nicht nur zu Kapitalflucht, sondern hat vernichtende Auswirkungen auf die Bilanzen.

Das Wechselkursregime ist daher einer der zentralen institutionellen Faktoren, um die Dynamik des finanz-dominierten Akkumulationsregimes zu verstehen. In Europa war die größte Änderung diesbezüglich

\footnotetext{
Siehe Duménil/Lévy (2001) und Stockhammer (2004b, Table 5.5). Änderungen in der Volkswirtschaftlichen Gesamtrechung machen eine Aktualisierung dieser Daten schwierig.

8 Auch bei rascher Erholung des Wirtschaftswachstums nach der Krise hinterlässt diese oft längerfristige Spuren. Onaran (2005) findet, dass die Einkommensverteilung oft nachhaltig verändert wurde.
} 


\section{Abb. 1: Nominelle Lohnstückkosten in der EU-12 (1994=100)}
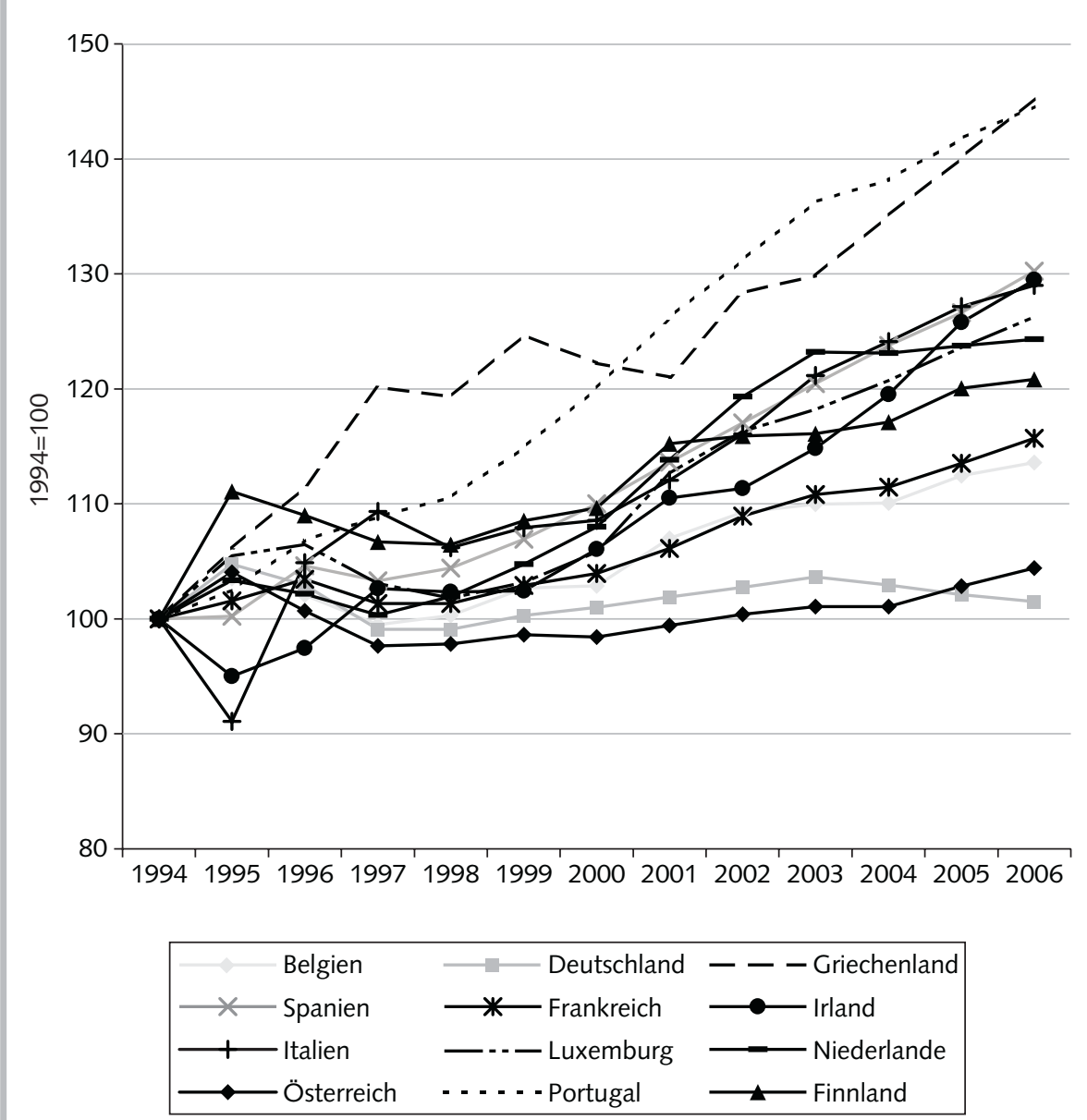

Quelle: Europäische Kommission 2007. Darstellung des Autors.

WSI MITTEILUNGeN

natürlich das Europäische Währungssystem (EWS) und die Einführung des Euro. Letztere war selbst auch eine Reaktion auf die EWS-Krise 1992/93, als das System fixer Wechselkurse zusammenbrach und etliche Währungen um $20 \%$ oder mehr abwertete. Auf den ersten Blick scheint das EuroSystem ein Erfolg gewesen zu sein. Die Märkte nahmen den Euro an und die Inflationsraten sanken auf niedrige Niveaus, was auch die Realzinsen in den früheren Hochinflationsländern deutlich gesenkt hat. Allerdings sind die Inflationsdifferentiale trotz der allgemein niedrigeren Infla- tionsraten bestehen geblieben, was zu einer schleichenden, aber beständigen Veränderung der realen Wechselkurse geführt hat. Abbildung 1 zeigt die Entwicklung der nominalen Lohnstückkosten seit der EuroEinführung. Deutschland hat demnach real um rund $20 \%$ im Vergleich zu Portugal, Irland und Spanien abgewertet, was sich auch in kräftigen Handelsbilanzungleichgewichten äußert. Für die kommenden Jahre stellt dies eine große Herausforderung für die Wirtschaftspolitik dar, da das Euro-System keine geeigneten Mechanismen hat, diese Ungleichgewichte abzu-

\begin{tabular}{|c|c|c|c|c|}
\hline & $1970 \mathrm{er}$ & $1980 \mathrm{er}$ & $1990 \mathrm{er}$ & $2000 \mathrm{er}$ \\
\hline Wachstum BIP, real & 3,27 & 2,43 & 2,14 & 1,49 \\
\hline Inflation (BIP-Deflator) & 9,21 & 6,13 & 2,41 & 2,01 \\
\hline langfristige Zinsen, real & 1,40 & 4,04 & 4,66 & 2,45 \\
\hline Arbeitslosenrate & 4,16 & 8,55 & 9,61 & 8,47 \\
\hline Wachstum Löhne, real & 3,23 & 0,62 & 1,19 & 0,45 \\
\hline
\end{tabular}

bauen. Zum Ausgleich müssten die mediterranen Länder (und Irland) über mehrere Jahre Inflationsraten deutlich unter der deutschen aufweisen. Da die deutsche Inflationsrate aber nahe Null ist, wäre dies nur bei Deflation möglich.

Europa hat also auf die Liberalisierung der Kapitalflüsse mit der Einführung einer gemeinsamen Währung reagiert und damit das Risiko von Währungskrisen (innerhalb Europas) beseitigt. Jedoch hat es damit auch den nominalen Wechselkurs als einen wichtigen Anpassungsmechanismus aufgegeben. Statt Wechselkurskrisen werden daher schleichende Ungleichgewichte und deflationäre Tendenzen die europäische Entwicklung bestimmen.

\section{Das finanz-dominierte Akkumulationsregime als Regime geringen Wachstums}

Wir können die bisherigen Ergebnisse nun kurz zusammenfassen. Für die europäischen Länder gibt es kaum Hinweise auf einen möglichen Konsumboom. Zwar hat die Verschuldung der Haushalte zugenommen, aber dies scheint gerade ausgereicht zu haben, um die negativen Effekte durch den Rückgang der Lohnquote zu kompensieren. Die Effekte der Finanzialisierung hohe Realzinsen, "Shareholder Value“Orientierung und gestiegene Unsicherheit - auf die Investitionsquote waren negativ, und die steigenden Profite führten nicht zu einem nennenswerten Anstieg der Investitionen. Insgesamt ist damit die makroökonomische Wachstumsdynamik enttäuschend. Tabelle 3 fasst wichtige Eckdaten für den Euro-Raum zusammen.

Das Wirtschafswachstum weist einen fallenden Trend über den gesamten Zeitraum auf. Lag es in den EU 12-Ländern in den 1970er Jahren noch bei mehr als $3 \%$ im Jahr, so sackte es in den 1990er Jahren auf rund 1,5\% ab. Parallel dazu gingen die Inflationsraten zurück, was erklärtes Ziel neoliberaler Politik war. Die realen Zinsen stiegen Anfang der 1980er Jahre deutlich an und fielen ab Mitte der 1990er Jahre im Zuge der Euro-Einführung (wobei dieser Fall vor allem für die mediterranen Länder gilt). Die realen Zinssätze sind auch in den 1990er und 2000er Jahren höher als das Wirtschaftswachstum, was den Bruch mit 
der keynesianischen Wirtschaftspolitik im Fordismus illustriert. Gleichzeitig sind die Arbeitslosenraten angestiegen, was - gemeinsam mit der Globalisierung - die Lohnentwicklung gehemmt hat. Seit den 1980er Jahren hinken die Löhne der Produktivität deutlich hinterher und die Lohnquote sinkt.

Das finanz-dominierte Akkumulationsregime ist damit als ein Regime des bescheidenen Wachstums zu charakterisieren. Auch wenn im Detail weiter zu belegen wäre, ob die Änderungen in den makroökonomischen Verhaltensfunktionen auf die Finanzialisierung zurückzuführen sind, so erscheint es aufgrund der vorliegenden Evidenz plausibel, dass sie wesentliche Impulse setzte.

Allerdings war das finanz-dominierte Akkumulationsregime in Europa bisher kaum von heftigen Wirtschaftskrisen gekennzeichnet, die von Finanzmärkten ausgingen, wie dies in Südost-Asien, Lateinamerika oder der Türkei der Fall war. Dies ist wohl auf zwei Faktoren zurückzuführen. Erstens wurde nach der EWS-Krise, die in einigen Ländern zu Rezessionen führte, das Euro-System etabliert, was Wechselkurskrisen innerhalb Europas ausschließt. Jedoch führt dieses System und der damit verbundene Stabilitäts- und Wachstumspakt zu eigenen Problemen, da Letzterer einen "deflationären bias" aufweist und außer Lohnbewegungen kaum Mechanismen zum Ausgleich von Ungleichgewichten vorhanden sind.

\section{Der Staatssektor: Groß trotz Neoliberalismus}

Ein zweiter Grund dafür, dass die Wirtschaftskrisen bisher nicht heftiger ausgefallen sind, liegt in der Größe des Staatssektors und seiner stabilisierenden Wirkung. Dies mag zunächst überraschend erscheinen, war doch die Reduktion des Staatseinflusses eines der Hauptziele des Neoliberalismus. Und tatsächlich kam es zu einer Welle der Deregulierung und der Privatisierung. Bemerkenswerterweise sind die Staatsquoten, die die direkten Staatsausgaben, nicht aber Unternehmen in Staatseigentum, umfassen, jedoch relativ stabil geblieben (Tabelle 4).

Nur in drei Ländern (Irland, VK und Niederlande) sind die Staatsquoten heute

\section{Tabelle 4: Staatsausgaben in \% des BIP (Durchschnitt)}

\begin{tabular}{lcccc}
\hline & $\mathbf{1 9 7 0 - 7 9}$ & $\mathbf{1 9 8 0 - 8 9}$ & $\mathbf{1 9 9 0 - 9 9}$ & $\mathbf{2 0 0 0 - 0 6}$ \\
\hline Österreich & 44 & 52 & 54 & 51 \\
Belgien & - & 56 & 52 & 50 \\
Dänemark & 45 & 56 & 58 & 54 \\
Finnland & 36 & 45 & 58 & 49 \\
Frankreich & - & - & 48 & 47 \\
Deutschland & 29 & 41 & 49 & 49 \\
Griechenland & 42 & 49 & 53 & 53 \\
Irland & 46 & 53 & 41 & 34 \\
Italien & 37 & 48 & 53 & 48 \\
Luxemburg & - & - & 40 & 41 \\
Niederlande & 48 & 56 & 50 & 46 \\
Portugal & 31 & 38 & 43 & 46 \\
Spanien & 26 & 39 & 44 & 57 \\
Schweden & 50 & 63 & 65 & 43 \\
Vereinigtes Königreich & 45 & 46 & 43 & 47 \\
EU & 40 & 36 & 50 & 36 \\
USA & 33 & 32 & 37 & 38 \\
Japan & 26 & & 35 & WSI MITTEILUNGEN \\
\hline & & & &
\end{tabular}

niedriger als in den 1970er Jahren. In den meisten Ländern stiegen sie bis in die frühen 1980er Jahre und stagnierten danach. Da der Neoliberalismus die Politik in den meisten Länder in diesem Zeitraum prägte, ist dies überraschend. Der Neoliberalismus war also bisher nur erfolgreich darin, den Anstieg der Staatsquoten zu reduzieren, nicht aber die Staatsquoten selbst. Da die Staatsausgaben über automatische Stabilisatoren eine stabilisierende Wirkung im Fall von Rezessionen ausüben, erklärt dies zum Teil, warum Krisen auf den Finanzmärkten in Europa und den USA bisher nicht so stark auf den realen Sektor übergegriffen haben.

Um Missverständnissen vorzubeugen: Dies bedeutet nicht, dass die neoliberale Hegemonie gar keine Auswirkungen auf die Staatsausgaben hatte. Erstens sind Privatisierungen in diesen Daten nicht erfasst, da Unternehmen in staatlichem Besitz, die nicht Teil der Hoheitsverwaltung sind, in der Volkswirtschaftlichen Gesamtrechung dem Privatsektor zugeschlagen werden. Zweitens kann sich bei gleich bleibendem Volumen die Struktur der Ausgaben verändert haben. Nicht zuletzt haben sich die Zinszahlungen des Staates durch die höheren Realzinsen deutlich erhöht. Im (ungewichteten) EU-Durchschnitt stiegen die Zinszahlungen von $0,7 \%$ (des BIP) in den 1970er Jahren auf 3,8 \% und 4,5\% in den 1980 er und 1990er Jahren und gingen seitdem leicht auf 2,5 \% zurück. Den stärksten Rückgang erfuhren dabei die mediterranen Länder.

\section{Ausblick}

Das finanz-dominierte Akkumulationsregime ist durch eine schwache Wachstumsdynamik gekennzeichnet. Europa und die USA wurden bisher noch nicht, wie die Schwellenländer Lateinamerikas und Asiens, von heftigen Krisen geschüttelt, die meist in Währungsturbulenzen ihren Ausgangspunkt hatten. Dies hat einerseits damit zu tun, dass trotz neoliberaler Attacken die Staatsquote nicht substanziell zurückgegangen ist und zur Stabilisierung der Konjunktur beitragen konnte, und andererseits damit, dass in Europa ein System fixer Wechselkurse etabliert wurde. Dieses System dämpft jedoch seinerseits das Wachstum und besitzt kaum Mechanismen zum Abbau interner Ungleichgewichte. Das finanz-dominierte Akkumulationsregime scheint weder in ökonomischen noch sozialen Dimensionen den Bedürfnissen nach einer allgemeinen Zunahme des Wohlstands nachzukommen. Fast drei Jahrzehnte neoliberal inspirierter Politik haben zu Massenarbeitslosigkeit und Polarisierung der Einkommensverteilung in Europa geführt. Nicht alles davon kann der Finanzialisierung angelastet werden, aber wie gezeigt wurde, sind negative Effekte klar spürbar. Eine deutliche Wende im wirtschaftspolitischen Regime wäre wünschenswert, ein zentraler Bestandteil wäre dabei eine Re-Regulierung des Finanzsektors. 
Aglietta, M. (1979): A Theory of Capitalist Regulation. The US Experience, London

Bank for International Settlements (BIS) (2005): 75th Annual Report, Basle

Bieler, A. (2003): European integration and eastward enlargement: the widening and deepening of neo-liberal restructuring in Europe. Queen's Papers on Europeanisation 8

Boone, L./Giorno, C./Richardson, P. (1998): Stock Market Fluctuations and Consumption Behaviour: Some Recent Evidence, OECD Economics Department Working Papers 208

Bowles, S./Gordon, D./Weisskopf, T. (1983): Beyond the Waste Land. A Democratic Alternative to Economic Decline, New York

Boyer, R. (1990): The Regulation School: A Critical Introduction, New York Boyer, R. (2000): Is a finance-led growth regime a viable alternative to Fordism? A preliminary analysis, in: Economy and Society 1, S. 111-145 Carruth, A./Dickerson, A./Henley, A. (2000): What do we know about investment under uncertainty?, in: Journal of Economic Surveys 2, S. 119-153

Case, K./Shiller, R./Quigley, J. (2001): Comparing Wealth Effects: The Stock Market Versus the Housing Market, NBER Working Paper W8606 Catte, P./Girouard, N./Price R./André C. (2004): Housing Markets, Wealth and the Business Cycle, OECD Economics Working Paper 394 Chirinko, R. (1993): Business fixed investment spending: modeling strategies, empirical results and policy implications, in: Journal of Economic Literature 31, S. 1875-1911

Crotty, J. (2003): The neoliberal paradox: the impact of destructive product market competition and impatient financial markets on nonfinancial corporations in the neoliberal era, in: Review of Radical Political Economics 3, S. 271-79

Dannreuther, C./Petit, P. (2006): Post-Fordism, beyond national models: the main challenges for regulation theory, in: Competition and Change 2, S. $100-112$

Duménil, G./Lévy, D (2001): Costs and benefits of Neoliberalism: a class analysis, in: Review of International Political Economy 4, S. 578-607 Epstein, G./Jayadev, A. (2005): The rise of rentier incomes in OECD countries: finanzialisation, central bank policy and labor solidarity, in: Epstein, G. (Hrsg.): Finanzialisation and the World Economy, Cheltenham, S. $46-74$

Europäische Kommission (2007): AMECO database,

http://ec.europa.eu/economy_finance/indicators/annual_macro_economic_database/ameco_en.htm

Ford, R./Poret, P. (1991): Business investment: recent performance and some implications for policy, in: OECD Economic Studies 16, S. 79-131

Girouard, N./Kennedy M./André, C. (2006): Has the Rise in Debt Made Households More Vulnerable?, OECD Economics Working Paper 535
Gordon D. M./Edwards R./Reich M. (1982): Segmented Work, Divided Workers. The Historical Transformation of Labour in the United States, Cambridge

Hein, E./Vogel, L. (2007): Distribution and Growth Reconsidered - Empirical Results for Austria, France, Germany, the Netherlands, the UK and the USA, IMK Working Paper 3

Hein, E./van Treeck, T. (2007): 'Financialization' in Kaleckian/PostKaleckian Models of Growth and Distribution and Growth, IMK Working Paper 7

Krippner, G. (2005): The financialization of the American economy, in: Socio-Economic Review 3, S. 173-208

Lazonick, W./O'Sullivan, M. (2000): Maximising shareholder value: a new ideology for corporate governance, in: Economy and Society 1 , S. $13-35$

Lipietz A. (1985): The Enchanted Word, London

McDonough, T./Nardone, E. (2006): Social structures of accumulation, the regulation approach and the European Union, in: Competition and Change 2, S. 200-212

Naastepad, R./Storm, S. (2006/7): OECD demand regimes (1960-2000), in: Journal of Post-Keynesian Economics 2, S. 213-248 OECD (2007): Economic Outlook database. www.sourceoecd.com Onaran, Ö. (2005): Life After Crisis for Labor and Capital in the Era of Neoliberal Globalization, Vienna University of Economics and Business Administration, Growth and Employment Working Paper 43

Schaberg, M. (1999): Globalization and the Erosion of National Financial Systems. Is Declining Autonomy Inevitable? Cheltenham, UK Skott, P./Ryoo, S. (2007): Macroeconomic Implications of Financialization, Univ. of Massachusetts Amherst Department of Economics Working Paper 2007-08

Stockhammer E. (2004a): Financialization and the slowdown of accumulation, in: Cambridge Journal of Economics 5, S. 719-741

Stockhammer, E. (2004b): The Rise of Unemployment in Europe:

A Keynesian Approach, Cheltenham

Stockhammer, E. (2005/06): Shareholder value-orientation and the investment-profit puzzle, in: Journal of Post Keynesian Economics 2, S. $193-216$

Stockhammer, E./Onaran, Ö./Ederer, S. (2007): Functional Income Distribution and Aggregate Demand in the Euro Area, Vienna University of Economics and Business Administration, Department of Economics Working Paper Series 102

Van Apeldoorn, B. (1999): Transnationalization and the restructuring of Europe's socioeconomic order, in: International Journal of Political Economy 1 , S. $12-53$ 